\title{
Proprioceptive integration and body representation: insights into dancers' expertise
}

\author{
Corinne Jola ${ }^{1,2)}$, Angharad Davis ${ }^{3)}$, Patrick Haggard ${ }^{2)}$ \\ 1) Psychology Department, University of Surrey, UK \\ 2) Institute of Cognitive Neuroscience, University College London, UK \\ ${ }^{3)}$ Royal Free and University College Medical School London, UK
}

Correspondence concerning this article should be addressed to:

Dr. C. Jola

Psychology Department

University of Surrey

Guildford

GU2 7XH

Tel: (01483) 689432

E-mail: C.Jola@surrey.ac.uk

Running head: Proprioceptive body representation

Keywords: Proprioception, Multisensory, Body representation, Dance 
Abstract

The experience of the body as a single coherent whole is based on multiple local sensory signals, integrated across different sensory modalities. We investigated how local information is integrated to form a single body representation and also compared the contribution of proprioceptive and visual information both in expert dancers and non-dancer controls. A number of previous studies have focused on individual differences in proprioceptive acuity at single joints and reported inconsistent findings. We used the established endpoint position matching task to measure absolute and directional errors in matching the position of one hand with the other. The matching performance was tested in three different conditions, which involved different information about the target position: only proprioceptive information from a 'target' hand which could be either the left or right, only visual information, or both proprioceptive and visual information. Differences in matching errors between these sensory conditions suggested that dancers show better integration of local proprioceptive signals than non-dancers. The dancers also relied more on proprioception when both proprioceptive and visual information about hand position were present. 
Introduction

Proprioception refers to the sense of position and movement of one's own body parts (see Sherrington 1907). Several classes of receptors contribute to proprioception, including muscle, joint, and skin receptors. Muscle spindle afferents are the most studied class of proprioceptive receptors, and play a key role in limb position sense (for an overview see Gandevia 1996), though joint and cutaneous afferents are also important in fine proprioceptive resolution (Ferrell and Smith 1988). In any case, proprioception is a key source of the mental representation of the body (Graziano and Botvinick 2002).

Previous studies of proprioception have focused on local information about the position of a single body part. However, we sense our body as a coherent whole, and proprioceptive perception of body position in space can be both phenomenally clear and highly accurate. For example, if one hand is placed anywhere in reachable space, it is relatively easy for a healthy individual - with or without vision - to bring the other hand to the same position. In fact, several neural computations underlie this 'proprioceptive matching' process. First, local proprioceptive information about the angles of individual joints must be combined to compute the position of the hand in space (Wolpert and Ghahramani 2000). Second, proprioceptive information must be integrated across the target hand and matching hand (Swinnen and Wenderoth 2004). This bilateral integration could involve linking individual limb-specific representations to a representation of the whole body, based on a common "egocentre" (Loomis and Lederman 1986). Third, and most importantly, the proprioceptive information must be correctly 
integrated with other sensory modalities, notably vision, to provide coherent perception of the body as a whole. This study investigated these multisensory transformations by measuring effects of expertise in multi-joint integration across limbs under different sensory conditions.

To do this, we used a simple endpoint position matching task (Von Hofsten and Rösblad 1988; Haggard et al. 2000; Jones et al. 2010; Van Beers et al. 2002; Wann 1991) where subjects indicate the perceived position of one hand (the 'target hand') when it is placed at a target location on a horizontal surface by reaching with their other hand to the matching location from under the surface. Successful performance of this task in the absence of vision requires integrating proprioceptive information from several joints and muscles within the limb, to compute the position of the target hand in space. Further, the sensory information about the target can be systematically manipulated. For example, absolute matching errors in terms of the error vector are generally greater when subjects have proprioceptive information about the target location but cannot see it, compared with when they can see the target location but cannot touch/feel it. A small further improvement occurs when both visual and proprioceptive information about the target location are available (Van Beers et al. 2002; Haggard et al. 2000; Von Hofsten and Rösblad 1988). In addition, the spatial pattern of matching error depends on the kind of sensory information available about the target, and on whether any proprioceptive information about the target position comes from the left or right arm (c.f. Haggard et al. 2000). As the matching errors for the left and right target hands were similar in size but 
mirrored in direction, Haggard et al. (2000) suggested that this so-called 'hand bias effect' indicates that subjects do not represent their body limbs in space by one single unified representation but two separate frames of reference originating at each shoulder. These frames might be described as 'propriocentres'. The endpoint position matching task can therefore provide evidence about the accuracy of different sensory modalities, about integrating of position information across multiple joints and about the extent to which subjects represent their bodies as coherent wholes.

Between-group differences in endpoint position matching have been used to study development (Nardini et al. 2010), impairment (Mon-Williams et al. 1999; Wann 1991) and age-related decline (Goble et al. 2009). For example, using this particular task, Nardini et al. (2010) showed that cross-modal integration was developed much earlier than previously reported, specifically before the age of six. However, while previous studies have identified dancers (Kuni and Schmitt 2004; Ramsay and Riddoch 2001) and gymnasts (Lephart et al. 1996) as showing superior perception at single joints, to our knowledge superior multisensory representation of endpoint position has not yet been studied. Finally, a number of studies have investigated differences in matching acuity between right and left target hands by using similar tasks, to study effects of handedness (for a review see Goble and Brown 2008). Here we have used the endpoint matching task to investigate how the brain integrates proprioceptive information across several individual joints to compute the location of the hand in 
space. We have investigated the multisensory basis of this representation in both non-dancers and expert dancers.

To achieve excellence in a dance performance, dancers must have outstanding proprioceptive ability to represent limb position in space. Studies of proprioception in dancers have generally focussed on postural motor responses (Golomer and Dupui 2000), or local proprioception at single joints (see above), rather than on endpoint position and multisensory integration. Also, some studies investigating local proprioception in dancers' lower limbs have reported no effect (Schmitt et al. 2005) or even an inverse effect of training (Barrack et al. 1984). Hence, any proprioceptive expertise may be specific to the postures across multiple joints in which the person is trained, and may not transfer to other postures. We therefore investigated the perceived position of the hand in space in dancers and in non-dancers, in order to address the representation of the body in space.

Here, our interest in multisensory integration and superior proprioception prompts three specific assumptions. First, dancers should show superior proprioceptive acuity compared to non-dancers (Ramsay and Riddoch 2001), implying smaller errors when the matching is based on proprioception. Second, dancers should have a more coherent body representation than non-dancers, leading to more similar matching responses whichever arm is used for matching. Third, dancers should show a stronger weighting of proprioceptive signals in multisensory conditions, relative to non-dancers. 
Confirmation of these hypotheses could advance understanding of how local proprioceptive information is integrated into a whole representation of the body, and how proprioceptive information is fused with other senses in novices and experts. Evidence for differences between these groups could be important both for motor learning and rehabilitation.

\section{Methods}

\section{Subjects}

Twelve expert dancers (age range $=18-21$ years, $M=19.08, S D=1.0,10$ females, all right handed) and twelve healthy subjects with no formal dance experience and no other form of professional physical training (age range $=20$ 24 years, $M=21.75, S D=1.42,9$ females, 2 left handed) participated with local ethical committee consent, and without knowledge of the experimental hypotheses. Only subjects with no history of injury to the upper body took part in the study. Their handedness was determined from their reported (and observed) writing hand, which is the most unequivocal indicator of hand preference (Dragovic 2004). We did not address questions of hand dominance, as they were not part of our study, though we noted that exclusion of the two left-handed subjects did not change the pattern of results.

The dancers had had at least two years of daily dance training, both in classical ballet with a minimum of 7.5 hours a week, and in contemporary dance with a minimum of 20 hours a week. At the time of testing, all dancers were 
actively training at BA or MA level in recognised higher education dance schools, from which they were recruited.

\section{Apparatus and Procedure}

Subjects were seated comfortably in front of a horizontal table (height 120 $\mathrm{cm}$ ) made from thin Perspex sheet (Figure 1). The top of the Perspex was covered with black paper. Five target locations were marked on the table by 7 $\mathrm{mm}$-diameter raised paper discs. The targets were aligned on the left-right axis with $11.1 \mathrm{~cm}$ spacing between centres. The subject's sternum was aligned with the central target (sternum-target distance: $31 \mathrm{~cm}$ ). A Logitech Webcam camera mounted upon a small tripod was placed centrally under the Perspex sheet with the lens aligned horizontally and thus parallel to it, ensured by the use of a spirit level. The camera image was aligned with four fiducial points on each corner of the Perspex sheet before each experimental block, to ensure standardised capture of position information with $640 \times 480$ pixels. On each trial, an image was taken, showing the Perspex table from beneath, including the location of the subject's index finger of the matching hand as they matched the target location, and also points corresponding to the target locations on the upper surface of the table. To help identify the precise matching location by image analysis, we placed a small black marker on the subject's index fingernails of the matching hand. 


\section{Task and Design}

The experimental design was based on previous studies using the endpoint position matching task (Haggard et al. 2000; Wann 1991). Subjects matched one of the five different target locations on the upper surface of the table by making a pointing movement underneath the table to the estimated corresponding location. Subjects perceived the actual target location either proprioceptively, visually, or both, according to condition (Figure 2). Only the sensory information about the target location above the table was modified, while the motor constraints for the matching hand to match the target location of the target hand remained similar. The underside of the table was entirely smooth and thus carried no information about the target location.

Insert Figure 2 about here

Varying sensory information about target position was provided in three blocked conditions. In the proprioceptive:proprioceptive $(P: P)$ condition, subjects were blindfolded. The experimenter passively placed the subject's index finger of the target hand on one of the five targets above the table. Each subject thus received proprioceptive information about the position of the target, and used this to direct the matching movement under the table to place their fingertip immediately beneath the target. Once the estimated location was reached, the subject briefly held their matching finger in this location while their fingertip position was photographed. The subject then returned the target and matching 
hands to their lap. In the visual:proprioceptive $(\mathrm{V}: \mathrm{P})$ condition, the experimenter verbally identified a target by a number written next to the target. Subjects were asked to fixate the target throughout each trial, but not to touch it or place their target hand on the upper surface of the table. Thus, they had only visual information about the target location. Finally, in the visualproprioceptive:proprioceptive (VP:P) condition, subjects received multisensory information about target location, from both vision and proprioception throughout each trial. The experimenter announced the target number and subjects made visually-guided reaching movements to place their index finger of the target hand on the designated target. They then moved the matching hand to the corresponding location underneath the table. Both hands were returned to their lap between trials. Note that there were two important differences between the V:P and P:P conditions: the availability of visual position information, and the presence of an active movement to the target location. Here, we consider the availability of visual information about target position to be more important, since Jones et al. (2010) found no difference in proprioceptive target location estimates between active and passive locations of the target hand. Thus, the experimental design ensured that conditions differed in the sensory information about the target location (i.e. visual and/or proprioceptive), and not in the sensorimotor control of the matching hand. Specifically, the movement of the matching hand under the table was not experimentally modified across the three conditions, so cannot explain any differences in matching error according to the sensory information available about target position. Also, subjects were asked to match 
the target location in their own time, and to avoid gross variations in movement speed.

Both hands were tested in each sensory condition in counterbalanced order. Thus, we designated either the left or right hand as the target hand in a particular block. In the P:P and VP:P conditions, one hand served as the target hand and was placed on the target locations, while the other hand served as the matching hand. In the V:P condition there was no target hand, as subjects experienced the target only visually. Instructed target location was varied randomly, with each of the five target locations being repeated six times. Hence, each subject made a total of 180 matching attempts: 3 sensory conditions $x 2$ target hands $\times 5$ target locations $\times 6$ repetitions.

Analysis

Matching error was defined as the distance between the target point (above the table) and the subject's matching index finger location (underneath the table). The error between each matching location and instructed target was digitised (ImageJ v1.41: http://rsbweb.nih.gov/ij/) and judged by 2 independent raters. The second rater judged a subset of the data (480 trials). The two raters' measurements correlated 0.8 in the $\mathrm{X}$ dimension and 0.9 in the $\mathrm{Y}$ dimension (Pearson's r). Matching errors were then converted from pixels to millimetres based on the ratio of the distance between target points on the table top and their distance in the pictures for each subject. Absolute matching error was defined as the length of a vector between target and matching location. We also calculated 
the components of the error vector in the $X$ (frontoparallel, left-right) and $Y$ (anterioposterior, front-back) directions (Figure 2).

Mixed-design ANCOVA was used for statistical analysis. Age was included as a covariate, since the dancers were significantly younger than the nondancers (see subjects), $t(22)=5.32, P<.001$ (assumption of homogeneity of regression slopes was approved). The average time in seconds for each trial was $M=6.06, S D=0.67$, and did not differ between groups $(P>.1)$. We had, however, clear directional predictions regarding our three main research questions (see introduction) based on previous studies. Therefore, one-tailed tests were sometimes used. These are clearly identified and justified in the results section.

Analyses of proprioceptive acuity were based on absolute matching error (length of the error vector). For analysis of effects of target hand and body representation coherence, the $x$ - and $y$ - components of the constant error were analysed separately, since spatial matching errors can be described by Cartesian error components (e.g., Haggard et al. 2000). Finally, we investigated multisensory fusion by quantifying to what extent hand-specific biases were present in each sensory condition. For this, we modelled $x$ - and $y$ - matching errors separately as a function of the target hand and the five different target locations by means of regression analyses. 


\section{Results}

Matching accuracy

Absolute matching accuracy, defined as the mean length of the error vector for each group and condition, is shown in Figure 3. As can be seen, for both dancers and non-dancers, matching was least accurate in the $P: P$ condition, much more accurate in V:P and more accurate still in the multisensory VP:P condition, with means of $4.77 \mathrm{~cm}$ (SE 0.30), $2.82(0.23)$, and $2.16(0.14)$, respectively. There was a significant main effect of condition: $F(2,42)=5.24, P<$ .01. All post-hoc comparisons between sensory conditions were significant, $t(23)$ $=5.38$ for PP vs. VP; $t(23)=8.56$ for PP vs. VPP; $t(23)=4.03$ for VP vs. VPP, respectively (all $P<.01$ with Bonferroni correction at alpha 0.05).Thus, performance was best in VP:P followed by $V: P$, followed by $P: P$, corroborating previous studies.

----- Insert Figure 3 about here

The between-subjects factor group (dancers vs. non-dancers) was also significant, $F(1,21)=3.00, P<.05$ (one-tailed) with dancers showing lower mean error vectors than non-dancers, $2.72(0.32)$ vs. 3.78 (0.32). Most importantly, the interaction between group and sensory condition was also significant, $F(2,42)=7.26, P<.01$. Follow-up simple effects analyses to explore this interaction showed that differences between the groups were significant only 
for the $P: P$ condition, $F(1,21)=9.48, P<.01$ (Bonferroni corrected at alpha 0.05), but not for the V:P or VP:P conditions $(P>.6)$.

Body coherence: spatial pattern of matching errors

The spatial pattern of matching errors was compared between each combination of group, condition, and hand to identify biases in the perceived position of the target hand. The results of the analysis with mean constant errors in the $\mathrm{x}$ - and $\mathrm{y}$-directions are shown in Figure 4.

----- Insert Figure 4 about here

As visible in Figure 4, there were clear differences in target location estimation between the two target hands: in all but one condition, the right target hand was perceived as being shifted to the right and rotated clockwise, while the left hand, placed in identical target locations, was perceived as shifted leftward and rotated counterclockwise. That is, the biases of the two hands were mirrorsymmetric, producing a cross-over appearance in the graph of the matching attempts, the 'hand-bias'. Three aspects of this hand-bias are noticeable in Figure 4. First, the hand bias was most evident in the P:P condition, suggesting that it is an effect driven by proprioceptive information about the target. Second, the hand-bias was less prominent in dancers than in non-dancers, suggesting a more coherent and less limb-specific representation of target hand position for the dancers. And third, in the $\mathrm{V}: \mathrm{P}$ condition, the non-dancers' matching errors resulted in a bowing pattern of the estimated target positions in both hands, while 
the dancers showed a reduced version of the cross-over effect. To test the significance of these observations, we performed ANCOVAs on translation and rotation biases.

Matching responses in the $P: P$ condition seem to be a geometric transformation of the target locations, involving both a rotation and a translation (Figure 4). To capture this transformation, we fitted linear regressions to matching errors, and tested the resulting regression coefficients. Separate regressions were performed for each subject's $\mathrm{x}$ - and y-errors, and for each target hand, and sensory condition. The regression slope for y-errors measures the rotation bias that produces the cross-over effect in Figure 4, while the intercept for $\mathrm{x}$-errors measures the translational shift along the $\mathrm{x}$-axis towards the target hand. A mixed-design ANCOVA on the regression slope of the y-errors, with the factors of sensory condition $(P: P, V: P, V P: P)$, group (dancers vs. nondancers), and target hand (right vs. left) was used. Age was again entered as a covariate, but was not significant here $(P>.5)$. The ANCOVA showed a significant main effect of target hand, $F(1,22)=95.14, P<.001$, confirming the cross-over effect seen in Figure 4. There was an interaction between target hand and sensory condition, $F(1,22)=61.46, P<.001$, because the rotation bias was stronger in $\mathrm{P}: \mathrm{P}$ than the other conditions. There was a further three-way interaction with group, $F(2,44)=3.18, P=.05$, as dancers showed a reduced hand-bias (Full results are given in Supplementary Table 1). This three-way interaction depended critically on the $\mathrm{P}: \mathrm{P}$ condition: when the $\mathrm{P}: \mathrm{P}$ condition was 
omitted this interaction became non-significant $(P>.3)$. No other effects or interactions were significant.

----- Insert Table 1 about here

We applied the same ANCOVA design as above to the calculated intercept values of the x-error regressions (Supplementary Table 1). Age was again a non-significant covariate $(P>.1)$ The ANCOVA showed main effects of hand, $F(1,22)=69.72, P<.01$, and condition, $F(2,44)=7.65, P<.01$, a twoway interaction between these factors, $F(2,44)=43.16, P<.01$, and a further three-way interaction between hand, condition, and group, $F(2,44)=3.59, P<$ .05. Again, the three-way interaction on the intercept depended critically on the $\mathrm{P}: \mathrm{P}$ condition: omitting the $\mathrm{P}: \mathrm{P}$ condition made the interaction non-significant $(\mathrm{P}>$ .7) . No other effects or interactions were significant. These analyses show that the cross-over effect was proprioceptive in origin, and was significantly reduced in dancers relative to non-dancers. This suggests that the dancers had a more coherent body representation than non-dancers, in the sense of a reduced discrepancy between proprioceptively perceived positions of the two hands.

\section{Visual-proprioceptive integration}

In the VP:P condition, both visual and proprioceptive information about target position was available. Hence, the magnitude of hand bias in the VP:P condition may reveal the contribution of proprioception to perceive a position when vision and proprioception are both available. Therefore, the rotational bias 
in the multisensory VP:P condition (calculated as the difference between the $y$ regression slopes for the two hands), and the translational bias (calculated as the difference between $\mathrm{x}$-regression intercepts for the two hands) were expressed as a percentage of the bias in the P:P condition (Supplementary Table 1). The resulting percentage estimates described the contribution of proprioception to matching in the multisensory VP:P condition for each subject. Two non-dancers showed small P:P rotational biases, so that the distribution of percentage values was highly non-normal. Median values and non-parametric testing were therefore used.

For non-dancers, $23 \%$ of the $\mathrm{P}: \mathrm{P}$ rotation bias was present in the VP:P condition. Dancers showed $37 \%$ of the P:P rotation bias in the VP:P condition. Dancers thus relied more on proprioception than non-dancers in multisensory conditions. This confirms the impression given visually (Figure 4): dancers showed a stronger cross-over effect than non-dancers in the VP:P condition. We found similar results for the translational bias $(23 \%$ of the $P: P$ translational bias was present in the VP:P condition for non-dancers, $46 \%$ for dancers). The percentage values were compared between groups using the Mann-Whitney test. Since we already showed more reliable proprioceptive information in dancers than in non-dancers (see above) we predicted greater reliance on proprioception in dancers than non-dancers, based on models of multisensory fusion (Ernst and Banks 2002). Therefore, one-tailed tests were used. The proprioceptive contribution to performance in the multisensory VP:P condition was significantly 
greater in dancers than non-dancers for the rotation bias, $U=104, P(Z(U))<.05$ one-tailed, and for translation bias, $U=101, P(Z(U))<.05$ one-tailed.

\section{Discussion}

We investigated multisensory perception of the position of the hand in space by asking expert dancers and non-dancers to perform an endpoint position matching task. Our data confirmed all three original hypotheses: First, dancers showed smaller error vectors when matching the target location based on proprioception. Second, dancers' matching errors depended less on which hand was used as the target than those of non-dancers. Third, dancers weighted visual and proprioceptive information about target hand position differently from nondancers. Therefore, dancers are better able to integrate local proprioceptive information from multiple joints to represent the position of the hand in space, have increased integration of the two arms into a single coherent body representation, and rely less on vision relative to non-dancers.

Our results also replicated previous findings using the endpoint matching task. The acuity with which the target hand was perceived varied according to the sensory information about target position. Accuracy was best when subjects had both visual and proprioceptive information about target position, worst when they had only proprioceptive information, and intermediate when only visual information was available (Von Hofsten and Rösblad 1988; Wann 1991). Then, Xand y-directional matching errors produced a spatial error pattern that was specific to the target hand, particularly in the P:P condition when only 
proprioceptive information about target position was available. In that case, matching errors showed a distinctive cross-over effect in both groups (Haggard et al. 2000).. Thus, proprioception seems to be linked to a specific 'propriocentre' for each arm, perhaps corresponding to the shoulder of origin. Thus, no single 'egocentre' exists for proprioception, in contrast with vision (Mitson et al. 1976).

Due to extensive training, dancers differ from non-dancers in terms of how they perform certain movements. Notably, they show greater range of movement. Further, dancers maintain trunk stability while moving the limbs. However, these biomechanical factors cannot explain differences between our $P: P$ and VP:P conditions, which involve very similar bimanual limb postures and moves. Thus, while the general pattern of matching errors (cross-over effect) may indeed reflect biomechanical constraints, the multisensory effects observed here can not be explained by postural or biomechanical differences.

Our study showed evidence for several differences in body representation between expert dancers and non-dancers. We found proprioceptive representation to be more accurate in dancers than in non-dancers. This was predicted from previous studies (Lephart et al. 1996; Ramsay and Riddoch 2001). However, our result extends the finding beyond local proprioception at a single joint, as studied previously, to the multi-joint proprioception of the position of the hand in space. Analyses of hand bias showed that the dancers' body representations were less limb-specific than those of non-dancers: that is, for dancers, translational and rotational matching errors were reduced and thus, the perceived position of the left hand at a given target position was closer to the 
perceived position of the right hand at the same position than for non-dancers. We therefore suggest that the dancer's body is integrated to form a single, coherent body representation to a greater extent than the non-dancers. This greater coherence could have been acquired through dance training (see Marmeleira et al., 2009), or it could simply reflect the fact that individuals with a more "centred" body representation are more likely to become dancers. In any case, our results show that proprioceptive representations may differ between people, and that there are important inter-group differences in coherence of body representation.

Differences between the two arms in proprioceptive and motor performance have been investigated by a number of studies (e.g. Shabbott and Sainburg 2008). In a recent review, Goble and Brown (2008) suggested that preferred and non-preferred arms have complementary roles during motor performance (see also dynamic dominance hypothesis: Bagesteiro and Sainburg 2003). Specifically, most studies showed better performance when the left arm was used to match the position of the right hand than vice versa, suggesting that the dominant hand benefits from better proprioceptive information than the nondominant hand. These differences have also been explained in terms of direction specificity between left-right and right-left interhemispheric transfers, as well as in terms of gains in the respective proprioceptive sensory-motor loops (Adamo and Martin 2009). However, our study did not focus on differences in proprioceptive ability between the two arms, and we found similar but mirror-symmetric hand bias for both hands. This suggests that proprioceptive differences between the 
left and right arms are relatively minor, even if potentially neuropsychologically informative (Goble and Brown 2008). In dance, posture and movement of both limbs must be accurate. Strong integration between the two arms in dancers may help to compensate for any dominance effects. A full study of handedness would require a design that can specifically separate lateralisation from dominance, for instance by having equal numbers of left- and right-handed subjects.

Finally, our study addresses the question of multisensory representation of the body. Variance is typically used as a measure of reliability (Ernst and Banks 2002) for optimal integration. To measure multi-sensory integration across several joints, we have used instead the degree to which characteristic unimodal biases are present in a multisensory condition as an index of weighting in multisensory fusion. Using this novel approach, we found that $23 \%$ of the translational and rotational biases found in non-dancers' proprioceptive $P: P$ matching were present in multisensory VP:P matching. Interestingly, this value is numerically consistent with values derived using variance estimates (Ernst and Banks 2002). Expert dancers, who had more accurate proprioception overall, gave significantly stronger multisensory weightings to proprioception. This pattern of results is consistent with theories of optimal multisensory integration: many perceptual skills involve prioritising one critical information source in a complex multisensory environment. To our knowledge, this is the first report of systematic expertise effects in multisensory body perception, though differences in multisensory postural control in dancers (Golomer and Dupui 2000) and in 
audiovisual integration in musicians (Petrini et al. 2009a), have been reported previously.

One unexpected result was the presence of hand-specific cross-over biases in the $\mathrm{V}: \mathrm{P}$ for dancers but not non-dancers. This was surprising, given that the cross-over effect was clearly associated with proprioceptive representation of the target position, yet information about the target position was purely visual in this condition. However, it may be linked to the enhanced weighing of proprioception in dancers mentioned above: we speculate that dancers might have used 'kinesthetic imagery' (Fourkas et al. 2006) to imagine their 'target' hand at the viewed target location (even though it actually remained in their lap in this condition). The dancers could then use this kinesthetic image to guide the matching hand. Petrini et al. (2009b) suggested that musicians generate internal models of actions which they use to replace missing visual information. However, further research would be required to investigate whether this could explain the dancers' performance in our V:P condition.

To summarise, superior local proprioception in expert dancers has been reported before (Golomer and Dupui 2000; Lephart et al. 1996; Mouchnino et al. 1993; Ramsay and Riddoch 2001; Schmitt et al. 2005; Vuillerme et al. 2001). Here we show that this effect is not confined to local, single-joint proprioception, but is present at higher levels of body representation also. We showed that dancers differ from non-dancers on measures of superordinate levels of proprioceptive representation. Specifically, they integrate the proprioceptive signals from each limb into a single frame of reference more strongly than non- 
dancers. This more coherent representation of the body could be either a cause or an effect of their motor expertise.

Dancers also weighted proprioception more strongly than non-dancers in multisensory fusion. There is a lively, though largely anecdotal debate about whether the ubiquitous presence of mirrors in training environments does or does not assist limb position sense (Radell et al. 2003). One recent study (Shabbott and Sainburg 2010) indicates that visual feedback during proprioceptive training improves accuracy in a normal population. Our results suggest that trained dancers are relatively uninfluenced by visual information about hand position. Further studies might investigate whether performance on proprioceptive tests, and robustness of proprioception to conflicting visual information are good predictors of final performance levels in motor skill training, and whether proprioceptive integration improves with such training (Ashton-Miller et al. 2001; Sims and Morton 1998).

\section{Acknowledgements}

This research was supported by a researchers' fellowship to CJ 106258 from the Swiss National Foundation (current affiliation Department of Psychology, University of Surrey, UK). Additional support was provided by The Leverhulme Trust, Research Grant F/07/134/AO to PH. We thank Dr Laura Brown for help with the manuscript, and Roger Bunce for technical support. 


\section{References}

Adamo DE, Martin BJ (2009) Position sense asymmetry. Exp Brain Res 192:8795

Ashton-Miller JA, Wojtys EM, Huston LJ, Fry-Welch D (2001) Can proprioception really be improved by exercises? Knee Surg Sports Traumatol Arthrosc $9: 128-136$

Barrack RL, Skinner HB, Cook SD (1984) Proprioception of the knee joint. Paradoxical effect of training. Am J Phys Med 63:175-181

Bagesteiro LB, Sainburg RL (2003) Nondominant arm advantages in load compensation during rapid elbow joint movements. J Neurophysiol 90:1503-1513

Dragovic M (2004) Towards an improved measure of the Edinburgh Handedness Inventory: a one-factor congeneric measurement model using confirmatory factor analysis. Laterality 9:411-419

Ernst MM, Banks MS (2002) Humans integrate visual and haptic information in a statistically optimal fashion. Nature 415:429-433 
Fourkas AD, lonta S, Aglioti SM (2006) Influence of imagined posture and imagery modality on corticospinal excitability. Behav Brain Res 168:190196

Ferrell WR, Smith S (1988) Position sense at the proximal interphalangeal joint of the human index finger. J Physiol 399:49-61

Gandevia SC (1996) Kinesthesia: roles for afferent signals and motor commands.

In: Rowell LB, Shepherd JT (eds) Handbook of Physiology Section 12. Exercise: Regulation and Integration of Multiple Systems. Oxford University Press, Oxford, pp 128-172

Goble DJ, Coxon JP, Wenderoth N, Van Impe A, Swinnen SS (2009) Proprioceptive sensibility in the elderly: degeneration, functional consequences and plastic-adaptive processes. Neurosci Biobehav Rev $33: 271-278$

Goble DJ, Brown SH (2008) The biological and behavioral basis of upper limb asymmetries in sensorimotor performance. Neurosci Biobehav Rev $32: 598-610$

Golomer E, Dupui P (2000) Spectral analysis of adult dancers' sways: sex and interaction vision-proprioception. Int J Neurosci 105:15-26 
Graziano MSA, Botvinick MM (2002) How the brain represents the body: insights from neurophysiology and psychology. In: Prinz W, Hommel, B (eds) Common Mechanisms in Perception and Action: Attention and Performance XIX. Oxford University Press, Oxford, pp 136-157

Jones SAH, Cressman EK, Henriques DYP (2010) Proprioceptive localization of the left and right hands. Exp Brain Res 204:373-383

Haggard P, Newman C, Blundell J, Andrew H (2000) The perceived position of the hand in space. Percept Psychophys 62:363-377

Kuni B, Schmitt H (2004) Peak torque and proprioception at the ankle of dancers in professional training [Article in German]. Sportverletz Sportschaden 18:15-21

Lephart SM, Giraldo JL, Borsa PA, Fu FH (1996) Knee joint proprioception: a comparison between female intercollegiate gymnasts and controls. Knee Surg Sports Traumatol Arthrosc 4:121-124

Loomis JM, Lederman SJ (1986) Tactual perception. In: Boff K, Kaufman L, Thomas J (eds) Handbook of Perception and Human Performance, 2nd vol. Wiley, New York, pp 31-41 
Marmeleira JF, Pereira C, Cruz-Ferreira A, Fretes V, Pisco R, Fernandes OM (2009) Creative dance can enhance proprioception in older adults. J Sports Med Phys Fitness 4:480-485

Mitson L, Ono H, Barbeito R (1976) Three methods of measuring the location of the egocentre: their reliability, comparative locations and intercorrelations. Can J Psychol 30:1-8

Mon-Williams MA, Wann JP, Pascal E (1999) Visual-proprioceptive mapping in children with developmental coordination disorder. Dev Med Child Neurol $41: 247-254$

Mouchnino L, Aurenty R, Massion J, Pedotti A (1993) Is the trunk a reference frame for calculating leg position? Neuroreport 4:125-127

Nardini M, Begus M, Manning C, Mareschal D (2010) Integration for visual and proprioceptive cues to hand position in development. Perception 39:146

Petrini K, Dahl S, Rocchesso D, Waadeland CH, Avanzini F, Pruce A, Pollick FE (2009a) Multisensory integration of drumming actions: musical expertise affects perceived audiovisual asynchrony. Exp Brain Res 198: 339-352 
Petrini K, Russell M, Pollick FE (2009b) When knowing can replace seeing in audiovisual integration of actions. Cognition 110:432-439

Radell SA, Adame DD, Cole SP (2003) Effect of teaching with mirrors on ballet dance performance. Percept Motor Skills 97:960-964

Ramsay JR, Riddoch MJ (2001) Position-matching in the upper limb: professional ballet dancers perform with outstanding accuracy. Clin Rehabil 15:324330

Schmitt H, Kuni B, Sabo, D (2005) Influence of professional dance training on peak torque and proprioception at the ankle. Clin J Sport Med 15:331-339

Shabbott BA, Sainburg RL (2010) Learning a visuomotor rotation: simultaneous visual and proprioceptive information is crucial for visuomotor remapping. Exp Brain Res 203:75-87

Shabbott BA, Sainburg RL (2008) Differentiating between two models of motor lateralization. J Neurophysiol 100:565-575

Sherrington CS (1907) On the proprio-ceptive system, especially in its reflex aspect. Brain 29:467-482 
Sims K, Morton J (1998) Modelling the training effects of kinaesthetic acuity measurement in children. J Child Psychol Psychiatry 39:731-746

Swinnen SP, Wenderoth N (2004) Two hands, one brain: cognitive neuroscience of bimanual skill. Trends Cogn Sci 8:18-25

Van Beers RJ, Wolpert DM, Haggard P (2002) When feeling is more important than seeing in sensorimotor adaptation. Curr Biol 12:834-837

Von Hofsten C, Rösblad, (1988) The integration of sensory information in the development of precise manual pointing. Neuropsychologia 26:805-821

Vuillerme N, Teasdale N, Nougier V (2001) The effect of expertise in gymnastics on proprioceptive sensory integration in human subjects. Neurosci Lett $311: 73-76$

Wann JP (1991) The integrity of visual-proprioceptive mapping in cerebral palsy. Neuropsychologia 29:1095-2106

Wolpert DM, Ghahramani Z (2000) Computational principles of movement neuroscience. Nat Neurosci 3:1212-1218 


\section{Figure Legends}

FIGURE 1: Experimental setup in the example of the VP:P condition. The target hand always points to target locations on the upper surface of the table. Subjects attempt to match its location under the table by pointing to the estimated corresponding location with the matching hand. The table is shown transparent for clarity only.

FIGURE 2: Experimental conditions and computed matching error. From left to right: sensory condition $\mathrm{P}: \mathrm{P}$ (only proprioceptive information available from the target hand, in this case the right hand; subjects are blindfolded), V:P (only visual information about target location available; there is no target hand, and the nonmatching hand rests in the subject's lap), VP:P (multisensory visual and proprioceptive condition; the subject places their hand on the target location, and also visually fixates this location). Inset: $\mathrm{x}$ and $\mathrm{y}$ components of matching error. Absolute matching error is defined as the length of the vector between target and matching location.

FIGURE 3: Absolute matching error (estimated means and SE) for each group in each sensory condition.

FIGURE 4: Mean matching error (bias) in the $\mathrm{x}$ - and $\mathrm{y}$-directions at each target location (circles), for left (black squares and solid line) and right (grey squares 
and dashed line) target hands separately, for sensory conditions; P:P (top), V:P (middle) and VP:P (bottom). Note the strong cross-over pattern, particularly in the $\mathrm{P}: \mathrm{P}$ condition, and particularly for non-dancers. Note that in the $\mathrm{V}: \mathrm{P}$ condition, there is no target hand, as the subject sees but does not touch the target. However, for comparability with other conditions, the condition where the subject matches a visual target with their left hand is plotted with grey squares and a dotted line, as if for a right-hand target, and the condition where the subject matches a visual target with the right hand is plotted with black squares and a solid line as if for a left-hand target (see text). 
Figures

FIGURE 1.

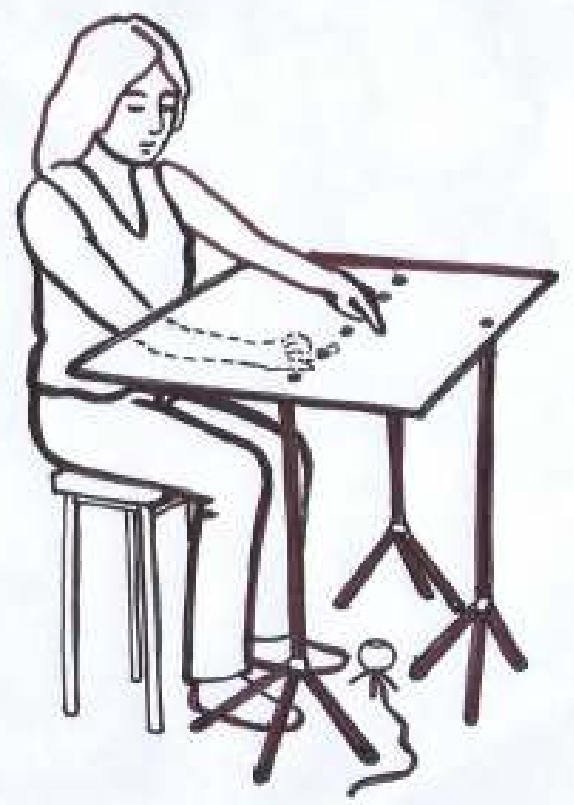

FIGURE 2.

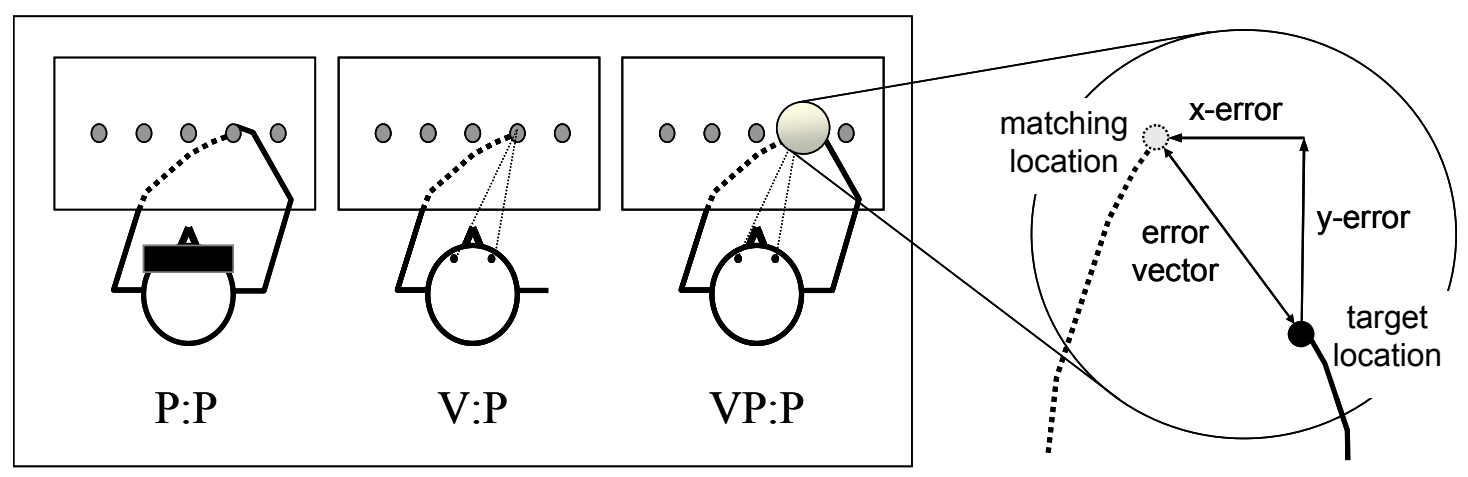


FIGURE 3.

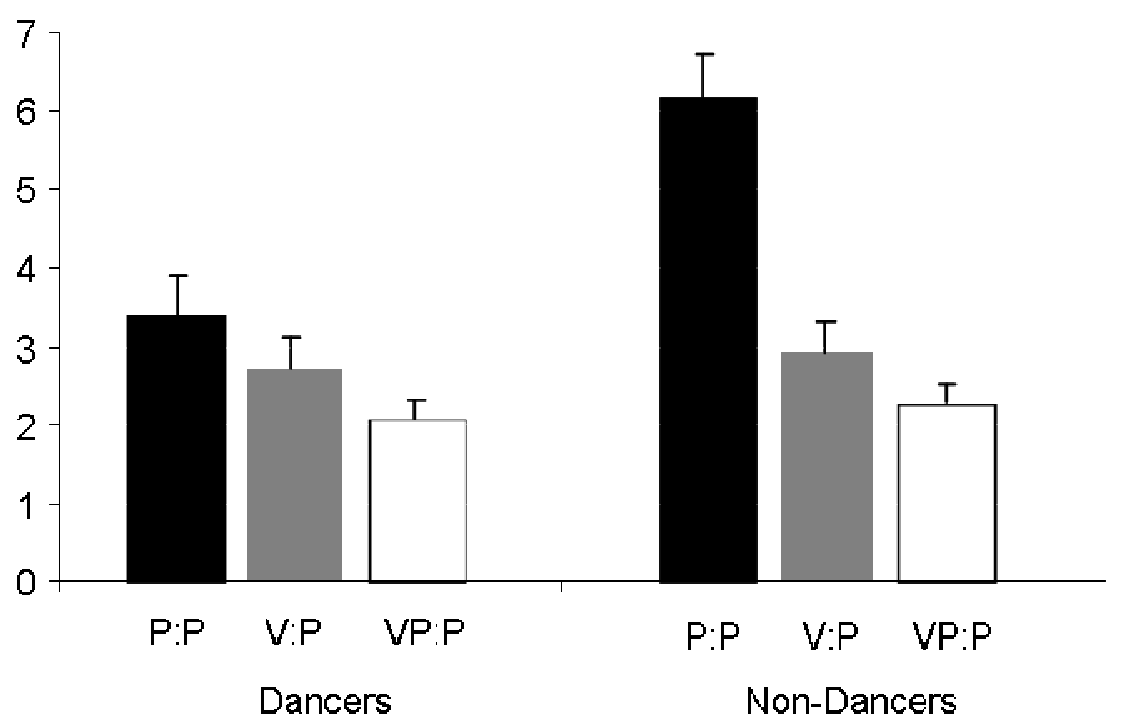


FIGURE 4.

Dancers
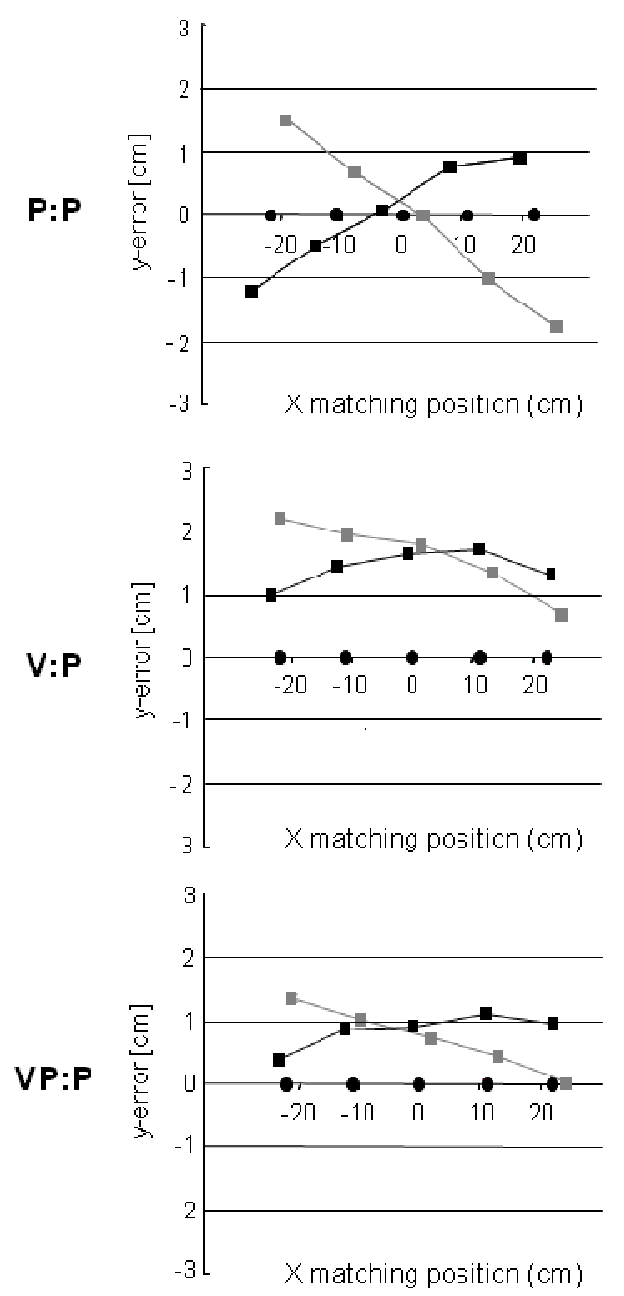

Non-Dancers

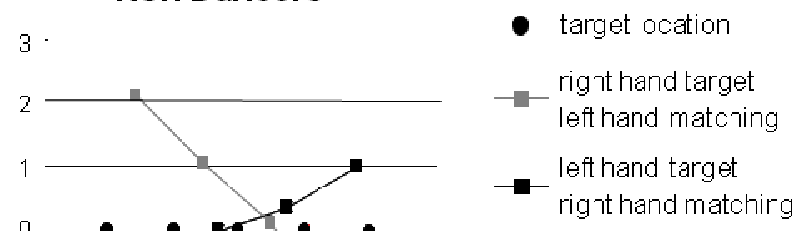

- 3 - x matchıng Fosition !cm)
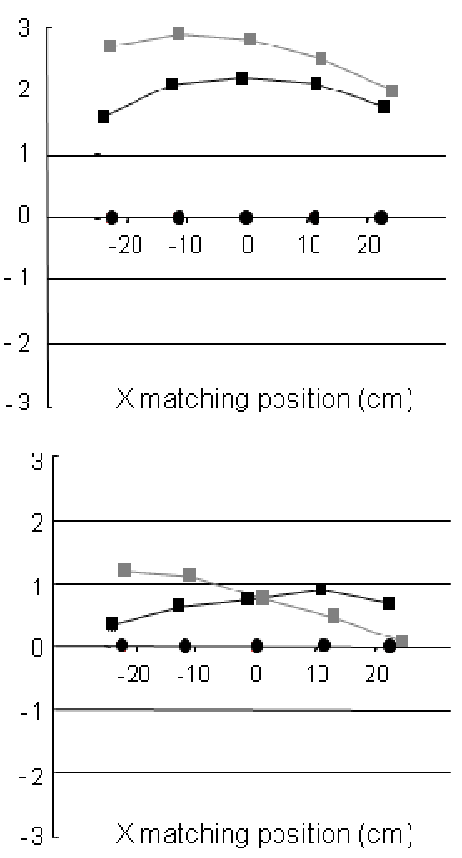